\title{
Highly discordant serology against Trypanosoma cruzi in central Veracruz, Mexico: role of the antigen used for diagnostic
}

\author{
Daniel Guzmán-Gómez ², Aracely López-Monteon 2,3 , María de la Soledad Lagunes-Castro 1,2, Carolina Álvarez-Martínez²,
} Manuel Jesús Hernández-Lutzon ${ }^{2}$, Eric Dumonteil ${ }^{4,5}$ and Angel Ramos-Ligonio ${ }^{2,3^{*}}$

\begin{abstract}
Background: Chagas disease is a parasitic disease caused by the protozoan parasite Trypanosoma cruzi. In Mexico, the burden of the disease is difficult to estimate and improving surveillance for Chagas disease is an important priority. We aimed here at determining the seroprevalence of $T$. cruzi infection in humans in a rural community in Veracruz.

Methods: Serum samples (196) were analyzed for T. cruzi infection using five enzyme-linked immunosorbent assay (ELISA) tests: two in-house tests based on crude parasite extract and three commercial ELISA kits. Because of highly discordant results, we further explored the importance of parasite antigens and strains by western-blot analysis.

Results: A total of 74 samples (37.7 \%) were reactive with at least one ELISA, but discordance among tests was very high. The best agreement was between Chagatest recombinant and Chagatek ELISA (Kappa index $=0.798$ ). The agreement between other combinations of tests ranged from 0.038 to 0.518 . Discordant samples were confirmed by western-blot analysis using up to nine parasite strains, giving a seroprevalence of $33.7 \%$.

Conclusions: Commercial tests had a very limited ability to detect T. cruzi infection in the study population. In-house tests based on crude parasite antigens showed a greater sensitivity but were still unable to detect all cases of T. cruzi infection, even when based on a local parasite strain. The high seroprevalence confirmed the hyper-endemicity of T. cruzi infection in the region. Reliable epidemiological surveillance of Chagas disease will require the development of improved diagnostic tests.
\end{abstract}

Keywords: Chagas disease, Serodiagnostic, ELISA, Antigen, Antibodies

\section{Background}

Chagas disease is a parasitic disease caused by the protozoan parasite Trypanosoma cruzi and transmitted primarily by hematophagous triatomine bugs. The disease is a major public health problem, affecting 8-10 million persons worldwide for a disease burden of over 29 million disability-adjusted life-years (DALYs), and a global health care cost reaching $\$ 627$ billion [1, 2]. The disease in endemic in the Americas, where vector species are widespread [3], but it is also becoming globalized due to

\footnotetext{
* Correspondence: angramos@uv.mx

²LADISER Inmunología y Biología Molecular, Facultad de Ciencias Químicas,

Universidad Veracruzana, Orizaba, Veracruz, Mexico

${ }^{3}$ Centro de Investigaciones Biomédicas, Universidad Veracruzana, Xalapa,

Veracruz, Mexico

Full list of author information is available at the end of the article
}

international migrations [4]. In Mexico, estimates indicate that there are at least 1-2 million infected persons, but possibly up to 6 million, although $T$. cruzi infection remains highly underreported $[5,6]$ and the true burden of the disease is difficult to estimate. Updating and improving surveillance and data for Chagas disease in Mexico should thus be an important priority, and help improve access to treatment for Mexican patients [7].

Serologic diagnostic for $T$. cruzi infection has undergone great progress in recent years, with the development and commercialization of many assays based on different parasite antigens. Indeed, there has been a shift in recent years from whole parasite extracts to more defined recombinant antigen mixtures showing better specificity [8-10]. The reported sensitivity and specificity 
of most of these assays appears very high (often $>98 \%$ for both). Nonetheless, their actual performance has been found to be somewhat lower [11] and no goldstandard has been identified for an accurate and reliable diagnostic of $T$. cruzi infection. As a consequence, the World Health Organization and most National guidelines in Latin American countries still recommend the use of two tests based on different principles and antigens, and in case of discordance, a third test can provide a final diagnostic.

Differences in test performance have often been attributed to the parasite stage used for antigen preparation, with amastigotes, epimastigotes and trypomastigotes potentially having a different antigenic profiles [12, 13]. Also, tests based on whole parasite lysate or crude antigens are believed to be much less reproducible and more difficult to standardize than those based on recombinant antigens [8-10]. The diversity of parasite strains has also been incriminated, leading to the recommendation that antigens from local strains should be favored to ensure optimum sensitivity and specificity of the tests. Indeed, T. cruzi has been divided into at least six discrete typing units (DTUs), referred to as TcI-TcVI, with very distinct characteristics [14]. Nonetheless, we previously found a good agreement between commercial tests, including a rapid test and a recombinant ELISA test, and an inhouse ELISA based on a crude extract from a local parasite strain from Mexico [15], suggesting that all these tests may be useful for the serodiagnostic of $T$. cruzi infection in Mexico [16]. ELISA tests based on a variety of TcI strains from Mexico and Guatemala and the CLBrener TcVI strain were found to perform similarly, suggesting that these strains were equally good sources of antigens for the detection of T. cruzi infection [17]. Indeed, agreement among tests is relatively high in most studies [18, 19], although the combination of tests implies that a small proportion of the samples will present discordant results, which are complicated to manage from a epidemiological and clinical point of view [19, 20]. Also, a low sensitivity of tests currently used can be a potential cause of underdiagnosis [11].

In this study, we aimed at determining the seroprevalence of $T$. cruzi infection in humans in a rural community in central Veracruz, using a combination of five ELISA tests based on different antigenic preparations. Due to highly discordant results, we further explored the possible role of parasite antigens and strains used in the serologic diagnostic of $T$. cruzi infection.

\section{Methods}

\section{Serum samples}

The study was carried out in the rural community of Las Josefinas, in central Veracruz, Mexico $\left(-96^{\circ} 41^{\prime} 31^{\prime \prime}\right.$ and $\left.18^{\circ} 28^{\prime} 26^{\prime \prime}\right)$. Information on Chagas disease and on the project was provided to the inhabitants of the community during open meetings organized in the rural medical unit of the community. Interested participants were given an appointment for themselves and their family to provide blood samples. The day of the appointment, written informed consent was obtained from each volunteer, and blood samples were collected in vacutainer tubes. Serum was separated by centrifugation at $1,200 \times \mathrm{g}$ for $10 \mathrm{~min}$ and samples were stored at $-70{ }^{\circ} \mathrm{C}$ until used. A total of 196 serum samples were collected and analyzed for $T$. cruzi infection using five different tests: two inhouse enzyme-linked immunosorbent assay (ELISA) based on crude parasite extract, three commercial ELISA diagnostic kits, and a western blot analysis.

\section{In-house ELISA}

Two in-house ELISA tests were used, based on epimastigote crude extracts of the CL-Brener (belonging to TcVI DTU) and LJ01 strains (TDIM/MEX/2014/LJ01/T. cruzi, a mixture of TcI and non-TcI DTUs, isolated from Triatoma dimidiata collected in the village of Las Josefinas, Veracruz, Mexico), respectively. Both strains were cultured in liver infusion tryptose medium supplemented with $10 \%(\mathrm{w} / \mathrm{v})$ fetal calf serum. Briefly, logarithmic phase parasites were harvested by centrifugation at $1,000 \times \mathrm{g}$ for $10 \mathrm{~min}$ at $4{ }^{\circ} \mathrm{C}$. The parasite pellet was suspended in $500 \mu \mathrm{L}$ of phosphate-buffered saline (PBS) (137 mM NaCl, $2.7 \mathrm{mM} \mathrm{KCl}, 4.3 \mathrm{mM} \mathrm{Na} 2 \mathrm{HPO}_{4}$, and 1.4 $\left.\mathrm{mM} \mathrm{KH}_{2} \mathrm{PO}_{4}, \mathrm{pH} 7.4\right)$ and lysed by cycles of freezing $\left(-70{ }^{\circ} \mathrm{C}\right)$ and thawing $\left(25^{\circ} \mathrm{C}\right)$. The suspension was centrifuged at $10,000 \times \mathrm{g}$ for $20 \mathrm{~min}$ at $4{ }^{\circ} \mathrm{C}$. The resulting supernatant (extract) was used as crude antigen extract. Protein concentration was determined by the Bradford method. In-house ELISA protocol was performed as follows, polystyrene plates (Costar Corporation, Cambridge, MA) were coated with the T. cruzi crude antigen extract $(10 \mu \mathrm{g} / \mathrm{mL})$ in carbonate buffer, $\mathrm{pH} 9.6$, and incubated overnight at $4{ }^{\circ} \mathrm{C}$. Unbound antigen was removed and plates were blocked with $200 \mu \mathrm{L}$ of PBS containing $5 \%$ weight/volume (w/v) non-fat milk for $2 \mathrm{~h}$ at $37{ }^{\circ} \mathrm{C}$. After washing with buffer (PBS with $0.05 \%$ w/v Tween 20), the plates were incubated with $50 \mu \mathrm{L}$ of serum samples (1:200 dilution with PBS); each sample was assayed in duplicate, and plates also included positive and negative control serum samples. Further washing steps were performed and a peroxidase labeled goat anti-human IgG antibody (Pierce, Rockford, IL) was added at a 1:5,000 dilution in PBS with $0.05 \% \mathrm{w} / \mathrm{v}$ Tween 20 and incubated for $1 \mathrm{~h}$ at room temperature. After eight washes, $100 \mu \mathrm{L}$ of 2,2,-azino-bis (3-ethylbenzthiazoline)-6-sulphonic acid (Zymed, San Francisco, CA) was added as substrate and the reaction was allowed to proceed for $20 \mathrm{~min}$ at room temperature. The reaction was stopped with $2 \% \mathrm{w} / \mathrm{v}$ sulfuric acid, and 
absorbance was read at $450 \mathrm{~nm}$ with an ELISA microplate reader (Multiscan MS; Labsystems, Vantaa, Finland).

\section{Commercial ELISA diagnostic tests}

We use three different commercial ELISA diagnostic kits, Chagatest ELISA recombinant v3.0 from Wiener lab (Rosario, Argentina) is based on six recombinant proteins (1, 2, 13, 30, 36 and SAPA antigens), reported to be conserved among strains. The manufacturer reports a sensitivity of $99.3-100 \%$ and a specificity of $98.7-100 \%$, and an agreement with other reference methods of $99.6 \%$. Chagatek ELISA from Lemos/Biomerieux (Santiago del Estero, Argentina) is based on purified T. cruzi antigens, and has a reported sensitivity of $100 \%$ and a specificity of $>99 \%$. The NovaLisa ${ }^{\circ}$ Chagas (Trypanosoma cruzi) IgG ELISA from NovaTec Inmunodiagnostica Gmbh (Dietzenbach, Germany) has a reported sensitivity and specificity of $>99 \%$, and is based on $T$. cruzi recombinant antigens. Assays were performed and cut-off determined according to the respective manufacturer's instructions.

\section{Western blot analysis}

Western blot was carried out as described before [21] using a panel of nine different $T$. cruzi strains from three DTUs. Briefly, $25 \mu \mathrm{g}$ of crude antigen extract from the MHOM/BR/1978/Sylvio-X10 (TcI DTU), CIET1, Camp8, Nayarit and H1 (all Tc I), MHOM/BR/1950/Y (TcII), TINF/CL/1945/Tulahuen (TcII), and TINF/BR/1963/ CL-Brener (TcVI) strains were separated by electrophoresis in a $10 \%$ sodium dodecyl sulfate-polyacrylamide gel, 4 replicates were performed and electroblotted onto a nitrocellulose membrane (Bio-Rad, Hercules, CA) at 80 volts at $4{ }^{\circ} \mathrm{C}$ for $1 \mathrm{~h}$. The membranes were blocked with $5 \% \mathrm{w} / \mathrm{v}$ solution of nonfat milk powder and washed with TBST buffer (50 mM Tris- $\mathrm{HCl}, \mathrm{pH} 7.4,150 \mathrm{mM} \mathrm{NaCl}$, $0.05 \%$ Tween 20). The nitrocellulose membrane was individually incubated $\left(2 \mathrm{~h}\right.$ at $\left.37^{\circ} \mathrm{C}\right)$ with $1 \mathrm{ml}$ of different human sera diluted 1:100 in TBST with $2 \%$ skim milk. Control membranes were incubated with positive and negative serum samples. Each membrane was washed three times with TBST and subsequently incubated with alkaline phosphatase-labeled goat anti-human IgG (Pierce). The membranes were then washed as above and the immune complexes were developed with nitrotetrazolium blue (NBT) and 5-bromo-4-chloro-3indolyl phosphate (BCIP). The reaction was stopped with water. Positive, negative, and secondary antibody controls were included in each experiment.

\section{Statistical analysis}

Cut-off OD values for commercial ELISA tests were determined as recommended by each manufacturer. For in house ELISA tests, the cut-off was defined as the mean
OD of negative serum samples $(n=20)$ plus three standard deviations. Frequencies of reactive samples were calculated for each ELISA tests. The agreement between different serological tests was assessed using the Kappa index. Seroprevalence rates were calculated for subgroups of the study population according to sex and age, as well as their $95 \%$ confidence intervals (CI). Differences among groups were assessed by $X^{2}$ tests.

\section{Results}

\section{ELISA assays}

A total of 196 blood samples were collected from inhabitants of the village of Las Josefinas, Veracruz, and tested with five ELISA tests for T. cruzi antibodies (Fig. 1). A total of $122(62.2 \%)$ samples were negative with all the tests, while $74(37.7 \%)$ were reactive with at least one test. However, discordance among tests was very high, with a total of at least 63/196 (32.1\%) samples with discordant ELISA results. Only one sample $(0.5 \%)$ was reactive with all five tests, and another one $(0.5 \%)$ with four of the five tests, and thus unambiguously considered as $T$. cruzi positives (Table 1). There were an additional nine samples $(4.5 \%)$ presenting reactivity in three ELISA tests, although with different combinations of tests. Eight samples $(4 \%)$ were reactive with NovaLisa ${ }^{\circ}$ Chagas IgG test, and the two ELISA with total extracts from the CL-Brener and LJ01 strains (Table 1), and one sample $(0.5 \%)$ was reactive with Chagatek ELISA (Laboratorio Lemos/Biomerieux), NovaLisa ${ }^{\circ}$ Chagas IgG (NovaTec Inmunodiagnostica), and the LJ01 total extract ELISA. A further 29 samples (14.8\%) were reactive with different combinations of two ELISA tests. Sixteen samples $(8.1 \%)$ were reactive with both in-house ELISA based on total T. cruzi extracts, but with none of the commercial tests, 12 samples $(6.1 \%)$ were reactive with NovaLisa $^{\circ}$ Chagas IgG test, and the total extract from

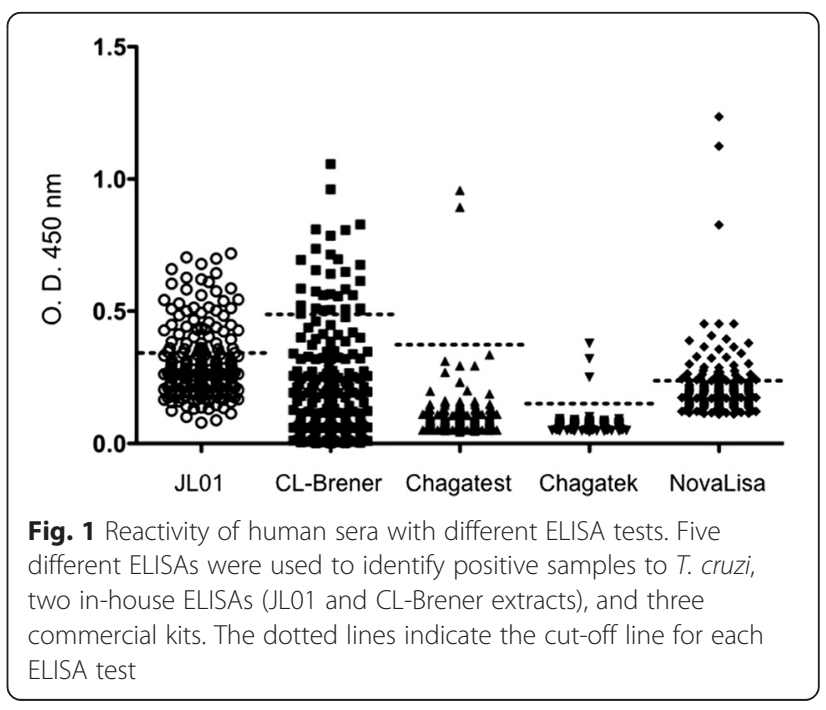


Table 1 Reactivity of samples against different test ELISA

\begin{tabular}{|c|c|c|c|c|c|c|}
\hline \multirow[t]{2}{*}{ Group } & \multirow[b]{2}{*}{ Ł01 } & \multirow[b]{2}{*}{ CL-Brener } & \multicolumn{3}{|l|}{ Tests } & \multirow{2}{*}{$\begin{array}{l}\text { Number of reactive } \\
\text { samples (\%) }\end{array}$} \\
\hline & & & NovaLisa & Chagatek & Chagatest & \\
\hline 1 & $\mathrm{R}$ & $R$ & $\mathrm{R}$ & $\mathrm{R}$ & $R$ & 1 (0.5 \%) \\
\hline 2 & $\mathrm{R}$ & $\mathrm{N}$ & $\mathrm{R}$ & $\mathrm{R}$ & $\mathrm{R}$ & 1 (0.5 \%) \\
\hline 3 & $\mathrm{R}$ & R & R & $\mathrm{N}$ & $\mathrm{N}$ & 8 (4.0\%) \\
\hline 4 & $\mathrm{R}$ & N & R & R & $\mathrm{N}$ & 1 (0.5 \%) \\
\hline 5 & $\mathrm{R}$ & R & $\mathrm{N}$ & $N$ & $\mathrm{~N}$ & 16 (8.1\%) \\
\hline 6 & $\mathrm{R}$ & N & R & $\mathrm{N}$ & $\mathrm{N}$ & 12 (6.1\%) \\
\hline 7 & $N$ & R & R & $\mathrm{N}$ & $\mathrm{N}$ & 1 (0.5 \%) \\
\hline 8 & $\mathrm{R}$ & N & $\mathrm{N}$ & $\mathrm{N}$ & $\mathrm{N}$ & 23 (11.7 \%) \\
\hline 9 & $N$ & N & R & $\mathrm{N}$ & $\mathrm{N}$ & 9 (4.6\%) \\
\hline 10 & $N$ & R & $\mathrm{N}$ & $\mathrm{N}$ & $\mathrm{N}$ & $2(1.0 \%)$ \\
\hline 11 & $N$ & N & $\mathrm{N}$ & $\mathrm{N}$ & $\mathrm{N}$ & $122(62.2 \%)$ \\
\hline Total $\left.\right|^{a}$ & 62 (31.6 \%) & 28 (14.3\%) & 33 (16.8 \%) & $3(1.5 \%)$ & $2(1.0 \%)$ & 196 (100\%) \\
\hline
\end{tabular}

Note: The sum of the values and percentages $\left({ }^{a}\right)$ of the last row does not reflect the total number of samples, but refers to the samples which were reactive for each test

Groups 1-4: Samples reactive for more than three tests

Groups 5-7: Samples reactive for two tests

Groups 8-10: Samples reactive for one test

Group 11: Sample negative for all test

$R$ Reactive, $N$ Negative

the LJ01 strain, and one sample with NovaLisa ${ }^{\circ}$ Chagas IgG test, and the total extract from the CL-Brener strain. Finally, an additional 34 samples (17.3\%) were reactive with a single ELISA test, with 23 samples (11.7 \%) reactive with the in-house ELISA with the LJ01 strain, 9 samples (4.6\%) were reactive with the NovaLisa ${ }^{\circ}$ Chagas IgG test, and another 2 samples (1.0 \%) with the CL-Brener ELISA test only (Table 1).

Based on a minimum of two reactive tests, a total of 40 samples $(20.4 \%)$ could thus be considered positive. However, there was a very poor agreement among tests, as indicated by very low Kappa indexes (Table 2). Indeed, the best agreement was between Chagatest recombinant 3.0 and Chagatek ELISA, with a Kappa index reaching 0.798 . The agreement between other combinations of tests was very low, with Kappa indexes ranging from 0.038 to 0.518 . Also, it remained unclear whether

Table 2 Kappa index among serological tests

\begin{tabular}{llllll}
\hline & A & B & C & D & E \\
\hline A & - & 0.518 & 0.339 & 0.185 & 0.044 \\
B & & - & 0.205 & 0.038 & 0.049 \\
C & & & - & 0.143 & 0.097 \\
D & & & & - & 0.798 \\
E & & & & & - \\
\hline
\end{tabular}

A: Crude extract LJ01 strain ELISA

B: Crude extract CL-Brener ELISA

C: NovaLisa ${ }^{\circledR}$ Chagas IgG (NovaTec Inmunodiagnostica)

D: Chagatek ELISA (Laboratorio Lemos/Biomerieux)

E: Chagatest Recombinant 3.0 (Wiener) samples with a single reactive ELISA should be considered as false positive or not.

\section{Western blot assays}

Because discordant results from $T$. cruzi serological test are often attributed to antigenic differences among parasite strains/DTUs or among recombinant proteins, we tested several of the serum samples in western blot assays using a panel of $T$. cruzi crude antigens from several parasite strains and DTUs, including five TcI, two TcII and one TcVI strains. As expected, the serum from Subject J004, which was reactive with all five ELISA tests, showed a strong banding pattern indicating a reactivity to many T. cruzi proteins for this serum (Fig. 2). Importantly, while the banding pattern varied clearly among the $T$. cruzi strains, this sample was strongly reactive with all strains and DTUs tested. In addition, serum samples which presented reactivity with different combinations of ELISA tests (Subjects J105 and J008, Fig. 2) also resulted reactive with different band patterns against all strains tested. A serum sample that was reactive with a single ELISA test was similarly reactive against all T. cruzi strains tested (Subject J054, Fig. 2). In fact, the serum samples reactive with a single ELISA ( 9 reactive with $\mathrm{NovaLisa}^{\circ}$ Chagas IgG test, 11 with LJ01 in house ELISA, and two with CL-Brener ELISA, respectively) and that were tested by western blot resulted all reactive against both LJ01 and Tulahuen parasite extracts (Additional file 1: Figure S1). This clearly indicated that these samples were not false positives of their 


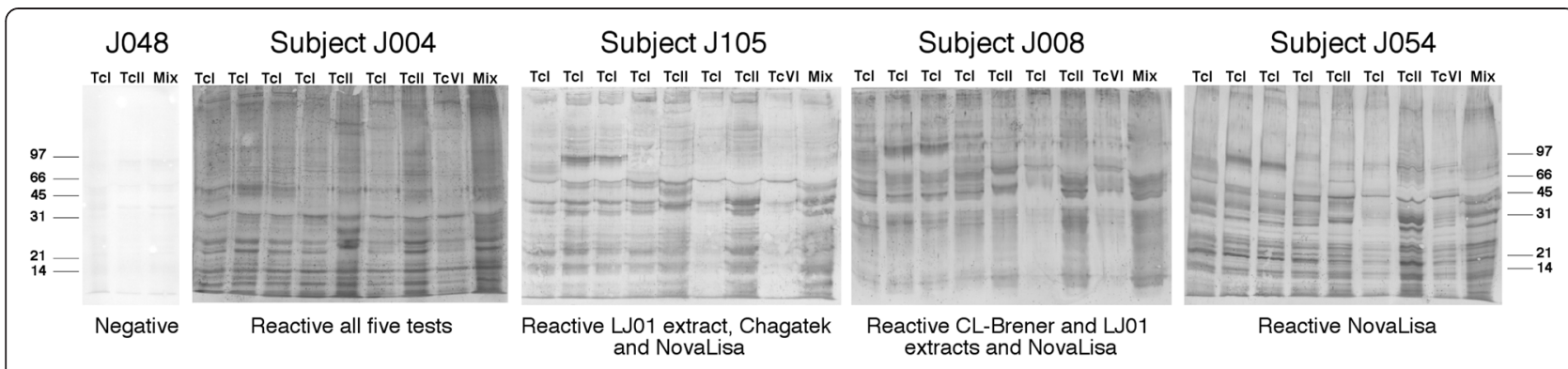

Fig. 2 Western blot analysis of serum samples against different T. cruzi strains and DTUs. Serum samples were tested for their reactivity against a panel of eight T. cruzi crude antigen from different strains and DTUs. These include, from left to right: Silvio-X10 (Tcl), Ciet-1 (Tcl), Camp-8 (Tcl), Nayarit (TCl), Y (TCll), H1 (TCl), Tulahuen (TCll), CL-Brener (TCVI), and LJ01 (Mixture of TCl and non-TCl)

respective ELISA tests but true positives for T. cruzi antibodies.

\section{Seroprevalence in the study population}

Based on the combination of ELISA tests and westernblot confirmation, a total of 62/184 samples (33.7\%; $95 \% \mathrm{CI}[27.3 ; 40.8]$ ) could thus be confirmed as positive with at least two tests. Men presented a significantly higher seroprevalence than women (55 vs $32 \%, X^{2}=$ 5.63, $P=0.017)$. Although seroprevalence tended to increase with age, this was not statistically significant $(36.8$ vs $40.8 \%$ for children $<12$ years old and $>12$ years old subjects, $\left.X^{2}=0.16, P=0.68\right)$, indicating a very active recent transmission of $T$. cruzi in this population.

\section{Discussion}

We evaluated here the seroprevalence of $T$. cruzi infection in humans in a rural community in Veracruz, using a combination of five ELISA tests based on different antigenic preparations. Unexpectedly, we found a very high level of discordance among the ELISA tests used, with very poor agreement among them. The seroprevalence varied from 1-1.5\% with two of the commercial ELISA up to $31.6 \%$ with an in-house ELISA based on a crude parasite extract from a local T. cruzi strain. Up to $32 \%$ of the samples presented discordant results among the ELISA tests and could thus be considered as inconclusive. This is contrary to most studies, which show a relatively good concordance among tests, and only a $1-2 \%$ of inconclusive results $[18,19]$. However, a study in Brazil also reported a high discordance among hemaglutination, immunofluorescence and ELISA tests [22].

The reactivity of samples by western blot confirmed that commercial ELISAs used in our study were very poorly sensitive for the detection of antibodies against $T$. cruzi in the study population. This is contrary to previous studies in Brazil, which showed that Chagatek ELISA had a good sensitivity and specificity [19, 23-25]. Similarly, Recombinant Chagastest ELISA also performed well in Argentina [18], Colombia [26], Brazil [19, 24, 25], and southern Mexico [15, 16]. We found here that the most sensitive commercial test was NovaLisa ${ }^{\circ}$ Chagas IgG test, detecting a seroprevalence of up to $16.3 \%$, but this still represented only half of the total seropositive cases confirmed by western blot. This test has not been used extensively so far, but seemed to perform very well in Colombia [27]. In particular, it presented very limited cross-reaction (1/7 serum samples) using sera from Leishmaniasis patients [27].

The differences in performance among commercial tests may be due to the number and nature of the antigen used, and a larger mixture of antigens may be more reliable than a few recombinant antigens $[18,28]$. This may also explain why crude parasite lysates, presenting a very large number of parasite antigens, can be more sensitive than recombinant antigens [29]. Indeed, the in-house ELISA tests based on the CL Brener and a local $T$. cruzi strain were able to detect the largest number of reactive serum samples, which were confirmed by western blot. However, an important drawback of such crude antigens is their insufficient specificity due to potential cross-reactivity of sera from leishmaniasis patients [29], which has motivated the development of test based on recombinant antigens. In our case, serum samples that were reactive with in house ELISAS are very unlikely to be from leishmaniasis patients. Indeed, although leishmaniasis is known to occur in the state of Veracruz [30], very few cases have been reported in the region where this study was performed. In addition, the Chagatek ELISA test was previously found to effectively react with serum from leishmaniasis patients (17/21 serum samples) [24], but gave very few reactive samples in our study population.

The low sensitivity of the commercial ELISA tests may also be due to a loss or masking of epitopes in the corresponding antigens due to glycosylation and other post-translational modifications. Indeed, carbohydrates epitopes have been found to be the target of a substantial part of host immune responses to other parasites 
[31, 32]. In the case of $T$. cruzi, a recombinant trypomastigote surface protein presented a low sensitivity for the serologic diagnostic of infection when compared to an indirect immunofluorescence assay [33], which may indeed be due to a lack of post-translational modifications of the protein produced in Escherichia coli. Glycosylation and hydroxylation have also been found to modulate the reactivity of T. cruzi glycosphingolipids fractions with human sera from Chagasic patients [34].

As previously, western blot was found to be a very good method for the confirmation of the reactivity of serum samples against T. cruzi antigens [35]. Importantly, it confirmed the reactivity of the serum samples against a wide variety of parasite strains, covering a large diversity of local DTUs, as well as strains from other geographic regions. Although the recognition pattern somewhat varied according to the strain used, all parasite extracts were clearly recognized by all serum samples tested. Nonetheless, western blot remains a test difficult to perform on large numbers of samples and for routine diagnostic/confirmation. In addition, only continuous epitopes are detected in western blots since proteins are denatured, while ELISAs rely on native proteins, which may result in a higher non-specific background. Tests based on T. cruzi excreted-secreted antigens (TESA) have also showed to be good for confirmation of inconclusive serologies [20, 24,36], but also share the limitation of been difficult to apply to large number of samples.

Improvement in current commercial ELISA tests is thus strongly needed. However, even the in-house ELISA test based on a local strain was unable to detect $11 \mathrm{~T}$. cruzi positive serum samples (5.6\%), which were detected with the CL-Brener crude antigen or the NovaLisa ${ }^{\circ}$ Chagas IgG tests. This suggested that the LJ01 parasite extract still did not include a repertoire of antigenic proteins that covered the entire $T$. cruzi diversity in the region. While the genetic diversity of T. cruzi was thought to be limited in Mexico, with TcI as the predominant DTU [37], several recent studies have found that other DTUs are also present and with high frequencies in both Mexico (TcII, TcIII, TcIV and TcV) and the United States (TcII) [38-40]. Thus, the identification and characterization of new parasite-specific antigens and epitopes [41-44] covering a wider range of the parasite antigenic profile may lead to the development of more specific and sensitive ELISA tests. Indeed, greater attention to parasite diversity should be considered for diagnostic development, similarly to what has been suggested for drug development [45]. Multiplex serology based on panels of novel recombinant proteins may provide an optimum diagnostic tool [46, 47], but need to be adapted to a test format that is easy to perform for the processing of large number of samples. In addition, the use of recombinant proteins expressed in eukaryotic systems, and thus with post-translational modifications, may improve their diagnostic accuracy.

Finally, the seroprevalence of $T$. cruzi infection we detected in the study population is very high, reaching $33.7 \%$. This confirmed our previous observations in this area, obtained with different ELISA tests [48]. Also, the continued presence of active transmission is evidenced by a high seroprevalence in children $<12$ years of age (36.8\%), also in agreement with a significant house infestation by Triatoma dimidiata and its frequent human feeding [49]. Thus, improved epidemiological surveillance and vector control interventions are urgently needed in the region.

\section{Conclusion}

In conclusion, we observed in this study a very high level of discordance among the ELISA tests used, with very poor agreement among them. Particularly, the commercial tests had a very limited ability to detect $T$. cruzi infection in the study population. In-house tests based on crude parasite antigens showed a greater sensitivity but were still unable to detect all cases of T. cruzi infection, even when based on a local parasite strain. Western-blot allowed the confirmation of an overall seroprevalence of $33.7 \%$, confirming the hyper-endemicity of $T$. cruzi infection in this region and the need for extensive epidemiological surveillance for a better prevention and control of Chagas disease. Nonetheless, this will require the development of improved diagnostic tests for an accurate detection of cases.

\section{Additional file}

Additional file 1: Figure S1. Western blot of serum samples against different $T$. cruzi strains. Serum samples reactive with a single ELISA test were confirmed by western blot using crude extract of the T. cruzi L01 (A) and Tulahuen (B) strains. These serum samples were reactive with the L01 ELISA (lanes 1-11), CL-Brener ELISA (lanes 12-13), and the NovaLisa ${ }^{\circ}$ ELISA (lanes 14-22). C+: positive control serum, C-: negative control serum. (JPEG $70 \mathrm{~kb}$ )

\section{Competing interests}

The authors declare that they have no competing interests.

\section{Author contributions}

DGG and MSLC collected samples; DGG, MSLC, CAM, and MJHL performed the experiments; ALM, ED and ARL designed the research. DGG, ALM, ED and $A R L$ wrote the manuscript and carried out data analysis. All authors contributed to the final interpretation of results and assisted in writing the manuscript. All authors read and approved the final manuscript.

\section{Acknowledgements}

We thank Dr. V. Monteon-Padilla for donating some of the strains used in this work. This work was supported by grant FOMIX CONACyT-Gobierno del Estado de Veracruz (VER-2008-C02-108783). 


\section{Author details}

'Doctorado en Ciencias Biomédicas, Universidad Veracruzana, Xalapa, Veracruz, Mexico. ${ }^{2}$ LADISER Inmunología y Biología Molecular, Facultad de Ciencias Químicas, Universidad Veracruzana, Orizaba, Veracruz, Mexico. ${ }^{3}$ Centro de Investigaciones Biomédicas, Universidad Veracruzana, Xalapa, Veracruz, Mexico. ${ }^{4}$ Laboratorio de Parasitología, Centro de Investigaciones Regionales "Dr. Hideyo Noguchi", Universidad Autónoma de Yucatán, Mérida, Yucatán, Mexico. ${ }^{5}$ Department of Tropical Medicine, Tulane University, School of Public Health and Tropical Medicine, New Orleans, LA, USA.

Received: 29 January 2015 Accepted: 4 September 2015

Published online: 17 September 2015

\section{References}

1. Lee BY, Bacon KM, Bottazzi ME, Hotez PJ. Global economic burden of Chagas disease: a computational simulation model. Lancet Infect Dis. 2013;13(4):342-8.

2. Hotez PJ, Bottazzi ME, Franco-Paredes C, Ault SK, Periago MR. The neglected tropical diseases of Latin America and the Caribbean: a review of disease burden and distribution and a roadmap for control and elimination. PLoS Negl Trop Dis. 2008;2(9):e300

3. Gourbière S, Dorn P, Tripet F, Dumonteil E. Genetics and evolution of triatomines: from phylogeny to vector control. Heredity. 2012;108:190-202.

4. Schmunis GA, Yadon ZE. Chagas disease: a Latin American health problem becoming a world health problem. Acta Trop. 2010;115(1-2):14-21.

5. Hotez PJ, Dumonteil E, Betancourt Cravioto M, Bottazzi ME, Tapia-Conyer R, Meymandi $\mathrm{S}$, et al. An unfolding tragedy of chagas disease in north america. PLoS Negl Trop Dis. 2013;7(10):e2300.

6. Dumonteil E. Update on Chagas' disease in Mexico. Salud Publica Mex 1999:41:322-7.

7. Manne JM, Snively CS, Ramsey JM, Salgado MO, Barnighausen T, Reich MR Barriers to treatment access for Chagas disease in Mexico. PLoS Negl Trop Dis. 2013;7(10):e2488

8. Camargo ME, Segura EL, Kagan IG, Souza JM, Carvalheiro Jda R, Yanovsky JF, et al. Three years of collaboration on the standardization of Chagas' disease serodiagnosis in the Americas: an appraisal. Bull Pan Am Health Organ. 1986;20(3):233-44

9. Umezawa ES, Bastos SF, Camargo ME, Yamauchi LM, Santos MR, Gonzalez A, et al. Evaluation of recombinant antigens for serodiagnosis of Chagas' disease in South and Central America. J Clin Microbiol. 1999;37(5):1554-60.

10. da Silveira JF, Umezawa ES, Luquetti AO. Chagas disease: recombinant Trypanosoma cruzi antigens for serological diagnosis. Trends Parasitol. 2001;17(6):286-91.

11. Afonso AM, Ebell MH, Tarleton RL. A systematic review of high quality diagnostic tests for Chagas disease. PLoS Negl Trop Dis. 2012;6(11):e1881.

12. Araujo FG, Guptill D. Use of antigen preparations of the amastigote stage of Trypanosoma cruzi in the serology of Chagas' disease. Am J Trop Med Hyg. 1984:33(3):362-71

13. Guimaraes MC, Celeste BJ, de Castilho EA, Mineo JR, Diniz JM. Immunoenzymatic assy (ELISA) in mucocutaneous leishmaniasis, kala-azar, and Chagas' disease: an epimastigote Trypanosoma cruzi antigen able to distinguish between anti-Trypanosoma and anti-Leishmania antibodies. Am J Trop Med Hyg. 1981;30(5):942-7.

14. Zingales B, Miles MA, Campbell DA, Tibayrenc M, Macedo AM, Teixeira MM, et al. The revised Trypanosoma cruzi subspecific nomenclature: rationale, epidemiological relevance and research applications. Infec Genet Evol. 2012;12(2):240-53

15. Gamboa-Leon R, Gonzalez-Ramirez C, Padilla-Raygoza N, Sosa-Estani S, Caamal-Kantun A, Buekens $P$, et al. Do commercial serologic tests for Trypanosoma cruzi infection detect Mexican strains in women and newborns? J Parasitol. 2011:97(2):338-43.

16. Gamboa-León R, Ramirez-Gonzalez C, Pacheco-Tucuch F, O'Shea M, Rosecrans K, Pippitt J, et al. Chagas disease among mothers and children in rural Mayan communities. Am J Trop Med Hyg. 2014:91(2):348-53.

17. Ballinas-Verdugo M, Reyes PA, Mejia-Dominguez A, Lopez R, Matta V, Monteon VM. Enzyme-Linked Immunosorbent Assay and Polymerase Chain Reaction Performance Using Mexican and Guatemalan Discrete Typing Unit I Strains of Trypanosoma cruzi. Vector Borne Zoonotic Dis. 2011;11(12):1569-75.
18. Vega Benedetti AF, Cimino RO, Cajal PS, Juarez Mdel V, Villalpando CA, Gil JF, et al. Performance of different Trypanosoma cruzi antigens in the diagnosis of Chagas disease in patients with American cutaneous leishmaniasis from a co-endemic region in Argentina. Trop Med Int Health 2013;18(9):1103-9.

19. Lapa JS, Saraiva RM, Hasslocher-Moreno AM, Georg I, Souza AS, Xavier SS, et al. Dealing with initial inconclusive serological results for chronic Chagas disease in clinical practice. Eur J Clin Microbiol Infec Dis. 2012;31(6):965-74.

20. Furucho CR, Umezawa ES, Almeida I, Freitas VL, Bezerra R, Nunes EV, et al. Inconclusive results in conventional serological screening for Chagas' disease in blood banks: evaluation of cellular and humoral response. Trop Med Int Health. 2008;13(12):1527-33.

21. Ramos-Ligonio A, Ramirez-Sanchez ME, Gonzalez-Hernandez JC, Rosales-Encina JL, Lopez-Monteon A. Prevalencia de anticuerpos contra Trypanosoma cruzi en donadores de sanger del IMSS, Orizaba, Veracruz, Mexico. Salud Publica Mex. 2006;48:13-21.

22. Souza RM, Amato Neto V. Discrepancies and consequences of indirect hemagglutination, indirect immunofluorescence and ELISA tests for the diagnosis of Chagas disease. Rev Inst Med Trop Sao Paulo. 2012;54(3):141-3.

23. Gilber SR, Alban SM, Gobor L, Bescrovaine Jde O, Myiazaki MI, Thomaz-Soccol $\checkmark$. Comparison of conventional serology and PCR methods for the routine diagnosis of Trypanosoma cruzi infection. Rev Soc Bras Med Trop. 2013:46(3):310-5.

24. Caballero ZC, Sousa OE, Marques WP, Saez-Alquezar A, Umezawa ES. Evaluation of serological tests to identify Trypanosoma cruzi infection in humans and determine cross-reactivity with Trypanosoma rangeli and Leishmania spp. Clin Vaccine Immunol. 2007;14(8):1045-9.

25. Araujo $A B$, Berne ME. Conventional serological performance in diagnosis of Chagas' disease in southern Brazil. Braz J Infect Dis. 2013;17(2):174-8.

26. Duarte LF, Florez O, Rincon G, Gonzalez Cl. Comparison of seven diagnostic tests to detect Trypanosoma cruzi infection in patients in chronic phase of Chagas disease. Colomb Med (Cali). 2014;45(2):61-6.

27. Llano M, Pavia P, Florez AC, Cuellar A, Gonzalez JM, Puerta C. [Preliminary evaluation of the commercial kit Chagas (Trypanosoma cruzi) IgG-ELISA (R) in Colombian individuals]. Biomedica. 2014;34(2):228-36.

28. Umezawa ES, Bastos SF, Coura JR, Levin MJ, Gonzalez A, Rangel-Aldao R, et al. An improved serodiagnostic test for Chagas' disease employing a mixture of Trypanosoma cruzi recombinant antigens. Transfusion. 2003;43(1):91-7.

29. Longhi SA, Brandariz SB, Lafon SO, Niborski LL, Luquetti AO, Schijman AG, et al. Evaluation of in-house ELISA using Trypanosoma cruzi lysate and recombinant antigens for diagnosis of Chagas disease and discrimination of its clinical forms. Am J Trop Med Hyg. 2012;87(2):267-71.

30. Gonzalez C, Rebollar-Tellez EA, Ibanez-Bernal S, Becker-Fauser I, Martinez-Meyer E, Peterson AT, et al. Current knowledge of Leishmania vectors in Mexico: how geographic distributions of species relate to transmission areas. Am J Trop Med Hyg. 2011:85(5):839-46.

31. Schallig HD, van Leeuwen MA. Carbohydrate epitopes on Haemonchus contortus antigens. Parasitol Res. 1996:82(1):38-42.

32. Harrison GB, Pulford HD, Doolin EE, Pernthaner A, Shoemaker CB, Hein WR. Antibodies to surface epitopes of the carbohydrate larval antigen CarLA are associated with passive protection in strongylid nematode challenge infections. Parasite Immunol. 2008:30(11-12):577-84.

33. Cetron MS, Hoff R, Kahn S, Eisen H, Van Voorhis WC. Evaluation of recombinant trypomastigote surface antigens of Trypanosoma cruzi in screening sera from a population in rural northeastern Brazil endemic for Chagas' disease. Acta Trop. 1992;50:259-66.

34. Villas-Boas MH, Wait R, Silva RB, Rodrigues ML, Barreto-Bergter E. Ceramide glycosylation and fatty acid hydroxylation influence serological reactivity in Trypanosoma cruzi glycosphingolipids. FEMS Microbiol Lett. 2005;244(1):47-52

35. Riera C, Verges $M$, Iniesta L, Fisa R, Gallego M, Tebar S, et al. Identification of a Western blot pattern for the specific diagnosis of Trypanosoma cruz infection in human sera. Am J Trop Med Hyg. 2012:86(3):412-6.

36. Zarate-Blades CR, Blades N, Nascimento MS, da Silveira JF, Umezawa ES. Diagnostic performance of tests based on Trypanosoma cruzi excreted-secreted antigens in an endemic area for Chagas' disease in Bolivia. Diagn Microbiol Infect Dis. 2007:57(2):229-32.

37. Bosseno MF, Barnabe C, Magallon Gastelum E, Lozano Kasten F, Ramsey J, Espinoza B, et al. Predominance of Trypanosoma cruzi lineage I in Mexico. J Clin Microbiol. 2002:40(2):627-32. 
38. Ramos-Ligonio A, Torres-Montero J, Lopez-Monteon A, Dumonteil E. Extensive diversity of Trypanosoma cruzi discrete typing units circulating in Triatoma dimidiata from central Veracruz, Mexico. Infect Genet Evol. 2012;12(7):1341-3.

39. Ibanez-Cervantes G, Martinez-Ibarra A, Nogueda-Torres B, Lopez-Orduna E, Alonso AL, Perea C, et al. Identification by Q-PCR of Trypanosoma cruzi lineage and determination of blood meal sources in triatomine gut samples in Mexico. Parasitol Int. 2013;62(1):36-43.

40. Herrera CP, Licon MH, Nation CS, Jameson SB, Wesson DM. Genotype diversity of Trypanosoma cruzi in small rodents and Triatoma sanguisuga from a rural area in New Orleans. Louisiana Parasit Vectors. 2015;8:123.

41. Mendes TA, Reis Cunha JL, de Almeida LR, Rodrigues Luiz GF, Lemos LD, dos Santos AR, et al. Identification of strain-specific B-cell epitopes in Trypanosoma cruzi using genome-scale epitope prediction and high-throughput immunoscreening with peptide arrays. PLoS Negl Trop Dis. 2013;7(10):e2524.

42. Reis-Cunha JL, Mendes TA, de Almeida LR, Ribeiro DR, Machado-de-Avila RA, de Oliveira TM, et al. Genome-wide screening and identification of new Trypanosoma cruzi antigens with potential application for chronic Chagas disease diagnosis. PLoS One. 2014;9(9):e106304.

43. Bottino CG, Gomes LP, Pereira JB, Coura JR, Provance Jr DW, De-Simone SG. Chagas disease-specific antigens: characterization of epitopes in CRA/FRA by synthetic peptide mapping and evaluation by ELISA-peptide assay. BMC Infect Dis. 2013;13:568.

44. Hernandez P, Heimann M, Riera C, Solano M, Santalla J, Luquetti AO, et al. Highly effective serodiagnosis for Chagas' disease. Clin Vaccine Immunol. 2010;17(10):1598-604.

45. Zingales B, Miles MA, Moraes CB, Luquetti A, Guhl F, Schijman AG, et al. Drug discovery for Chagas disease should consider Trypanosoma cruzi strain diversity. Mem Inst Oswaldo Cruz. 2014;109(6):828-33.

46. Cooley G, Etheridge RD, Boehlke C, Bundy B, Weatherly DB, Minning T, et al. High throughput selection of effective serodiagnostics for Trypanosoma cruzi infection. PLoS Negl Trop Dis. 2008;2(10):e316.

47. Viotti R, Vigliano C, Alvarez MG, Lococo B, Petti M, Bertocchi G, et al. Impact of aetiological treatment on conventional and multiplex serology in chronic Chagas disease. PLoS Negl Trop Dis. 2011;5(9):e1314.

48. Ramos-Ligonio A, López-Monteon A, Guzmán-Gómez D, Rosales-Encina JL, Limón-Flores $Y$, Dumonteil E. Identification of a hyper-endemic area for Trypanosoma cruzi infection in central Veracruz, Mexico. Am J Trop Med Hyg. 2010;83:164-70.

49. Torres-Montero J, López-Monteon A, Dumonteil E, Ramos-Ligonio A. House infestation dynamics and feeding sources of Triatoma dimidiata in central Veracruz, Mexico. Am J Trop Med Hyg. 2012;86(4):677-82.

\section{Submit your next manuscript to BioMed Central and take full advantage of:}

- Convenient online submission

- Thorough peer review

- No space constraints or color figure charges

- Immediate publication on acceptance

- Inclusion in PubMed, CAS, Scopus and Google Scholar

- Research which is freely available for redistribution 